\title{
Magnetic resonance imaging abnormalities with septic encephalopathy
}

\section{P F Finelli, D F Uphoff}

J Neurol Neurosurg Psychiatry 2004;75:1 189-1 191. doi: 10.1136/jnnp.2003.030833

A 48 year old woman, status post renal transplantation six years earlier, died after a two week illness characterised by fever, recurrent seizures, and coma. Widespread abnormalities were seen on neuroimaging. A diagnosis of septic encephalopathy was established on postmortem. We describe the magnetic resonance imaging findings of bilateral basal ganglia, thalamic, cerebellar, brainstem, and cerebral abnormalities in this patient, which correlate with the pathophysiology of septic encephalopathy.

E ncephalopathy in association with sepsis without evidence of hepatic or renal dysfunction, hypoxaemia, or other identifiable aetiology is referred to as septic encephalopathy (SE) and is often a diagnosis of exclusion. ${ }^{1}$ A neuroimaging pattern with neuropathology that corresponds with the pathophysiology associated with SE is described.

\section{CASE REPORT}

A 48 year old woman with hypertension and polycystic kidney disease, status post renal transplant six years earlier, was in good health until one week before admission, when she developed symptoms of urinary tract infection. A subsequent urine culture was positive for Escherichia coli. She was treated with ciprofloxacin; however, symptoms of haematuria, and nausea and vomiting developed, and she was admitted to hospital.

The patient was afebrile and her heart rate was 108/min, blood pressure 180/100 $\mathrm{mm} \mathrm{Hg}$, and respiratory rate 19/min. She was alert, oriented, and cooperative. The lungs were clear on auscultation, with no appreciable heart murmur. There was right lower quadrant tenderness. The rest of the physical examination was unremarkable. On hospital day 2, her temperature was $38.3^{\circ} \mathrm{C}\left(101{ }^{\circ} \mathrm{F}\right)$. She had a grand mal seizure of three to five minutes duration with an apnoeic spell of 30-40 seconds. Phenytoin ( $1 \mathrm{~g}$ ) was administered intravenously. A computed tomography (CT) scan of the head was negative. Later that day she had a second seizure, and she was intubated and transferred to the intensive care unit. Medications on admission included prednisone, mycophenolate, diltiazem, aciclovir, and hydrochlorothiazide. She was given levofloxacin $500 \mathrm{mg}$ intravenously followed by one dose of $250 \mathrm{mg}$ orally. Cefotaxime and ganciclovir were added on hospital day 4 .

On neurologic examination on day 2 the patient was lethargic with a non-focal examination. On day 3 she only opened her eyes to voice and pain, and on day 4 she was unresponsive even to deep pain. The extremities were flaccid and pupils were non-reactive. On day 3 she had another seizure followed by two further seizures on day 4 . Cerebrospinal fluid analysis on day 3 was unremarkable other than for protein $(195 \mathrm{mg} / \mathrm{dl})$. Repeat spinal fluid analysis on day 8 showed 235 white blood cells $/ \mathrm{mm}^{3}, 80 \%$ granulocytes, and protein $240 \mathrm{mg} / \mathrm{dl}$. Routine culture of the spinal fluid was negative. Polymerase chain reaction (PCR) for cytomegalovirus, herpes simplex virus, and Lyme's disease, and cryptococcal antigen and serological tests for Bartonella were all negative. A renal ultrasound and CT scan of the abdomen were normal. Blood and urine cultures were negative. A gallium scan on day 7 showed a focus of increased activity in the right lower quadrant. The electroencephalogram was diffusely slow with bilateral theta activity. Magnetic resonance imaging (MRI) on day 4 showed bilateral basal ganglia, cerebellum, brainstem, and temporal lobe abnormalities most prominent on the fluid attenuated inversion recovery (FLAIR) sequence (fig lA, B). Tl-weighted sequence showed a patchy decreased signal in the area corresponding to the abnormality on the FLAIR sequence, while diffusion-weighted images showed increased signals primarily in the basal ganglia. No definitive parenchymal enhancement was appreciable following gadolinium administration. The MR venography sequence was normal. Her attending physician noted no prolonged period of anoxia, and her blood pressure and oxygen saturation remained stable. The patient's condition continued to deteriorate, and on her thirteenth day in hospital the life support measures were withdrawn.

At autopsy, the native right kidney showed typical gross features of adult polycystic renal disease. There were multiple abscesses in the central portion of the kidney which on microscopy showed marked acute inflammation with neutrophils and fibrin. In one of these Gram staining and immunocytochemistry demonstrated the presence of E. coli. The transplanted kidney showed acute tubular necrosis.

Examination of the brain showed unremarkable major blood vessels without atherosclerosis. There was extensive infarction of the basal ganglia characterised by softening and slight discoloration of the caudate nucleus, putamen, and globus pallidus bilaterally without obvious gross involvement of the thalamus (fig 2A). Three relatively small foci of cortical infarction were also found, as well as infarction of the splenium of the corpus callosum. No gross abnormalities were apparent in the brain stem and cerebellum.

Microscopic examination of the grossly apparent infarcts showed necrotic lesions with marked acute inflammatory reaction, some of them verging on abscess formation; no organisms were identified with routine bacterial stains and immunochemistry. Additional small cortical infarcts not grossly apparent were also found. The basal ganglia showed large areas of infarction with acute inflammation. In both cortex and basal ganglia, small arteries were occluded by aggregates of polymorphs or by fibrin, while others showed striking fibrinoid necrosis (fig 2B, C). Despite the appearance

Abbreviations: $\mathrm{CT}$, computed tomography; FLAIR, fluid attenuated inversion recovery; MRI, magnetic resonance imaging; $\mathrm{SE}$, septic encephalopathy 

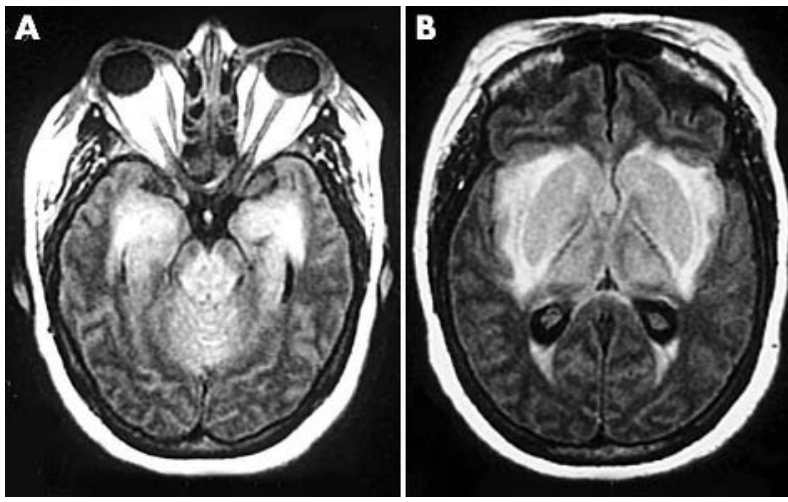

Figure 1 (A) Fluid attenuated inversion recovery (FLAIR) sequence with increased signals in the midbrain, vermis of cerebellum, and medial portions of both temporal lobes. (B) FLAIR sequence showing increased signals in the basal ganglia, thalamus, internal capsule, and adjacent white matter.

of septic infarction, no organisms were identified with B\&H (Brown \& Hopps) modification of tissue Gram stain or by immunocytochemical stain and PCR for E. coli. The thalamus showed evidence of oedema and scattered minute ischaemic lesions with macrophage reaction. Adjacent white matter tracts, including the internal capsule, also showed foci of axonal swellings. The hippocampi showed mild neuronal loss and acute neuronal ischaemic change. The brainstem showed evidence of oedema. Two blocks of medulla, although grossly normal, showed several minute lesions with axonal swelling and myelin breakdown with macrophage reaction (fig 2D). The cerebellum showed oedema of the white matter, acute ischaemic changes in numerous Purkinje cells and increased numbers of glial cells in the cortex.

\section{DISCUSSION}

SE is the most common form of encephalopathy in the medical intensive care unit ${ }^{2}$ and is probably underdiagnosed due to symptoms being masked by treatment such as sedation, mechanical ventilation, or neuromuscular blockade in critically ill patients. ${ }^{3}$ The encephalopathy is not thought to be a direct effect of the infecting organism and/or toxin, but rather that it arises from the action of inflammatory mediators on the brain or a cytotoxic response by brain cells to these mediators. ${ }^{3}$ This is supported by a recent post mortem study on four of 14 patients with SE in whom no microabscesses were found. ${ }^{2}$

The presence of a predisposition to sepsis, particularly urosepsis, in a patient with polycystic kidney disease, along with immunosuppression and prior urinary tract infection in the context of an unexplained encephalopathy and progressive febrile illness might have suggested the diagnosis of SE. Absence of positive blood or urine cultures and failure to identify a septic focus further complicated the diagnosis in our case. Despite appropriate antibiotic treatment on admission the patient worsened.

The pathophysiology of the systemic inflammatory reaction in SE is unclear, but it is most likely multifactorial and related to release of inflammatory mediators. A unique lipopolysaccharide, endotoxin, is pre-eminent among products implicated in the pathogenesis of sepsis and is well established as a trigger for the cellular and pathophysiological response to sepsis. Lipid A, a component of endotoxin, is a diglucosamine based phospholipid that induces a wide range of proinflammatory responses, including release of cytokines from macrophages and neutrophils, activation of the complement cascade, and upregulation of the adhesive capacity of neutrophils and endothelial cells. ${ }^{4}$ These factors result in reduced cerebral blood flow and oxygen extraction, cerebral oedema, disruption of the blood-brain barrier, impaired astrocyte function and neuronal death. ${ }^{13}$ Oxygen delivery to the brain is further limited by damaged erythrocytes from free radicals released by leucocytes. Coagulation abnormalities are a common feature of sepsis. Activation of the coagulation cascade and the subsequent deposition of fibrin can impair organ function by microvascular thromboses. Furthermore, products of coagulation are chemotactic for neutrophils and monocytes and hence may potentiate inflammatory processes. ${ }^{4}$

Published findings of MRI in SE are limited to a single report of non-specific white matter changes. ${ }^{5}$ The MRI
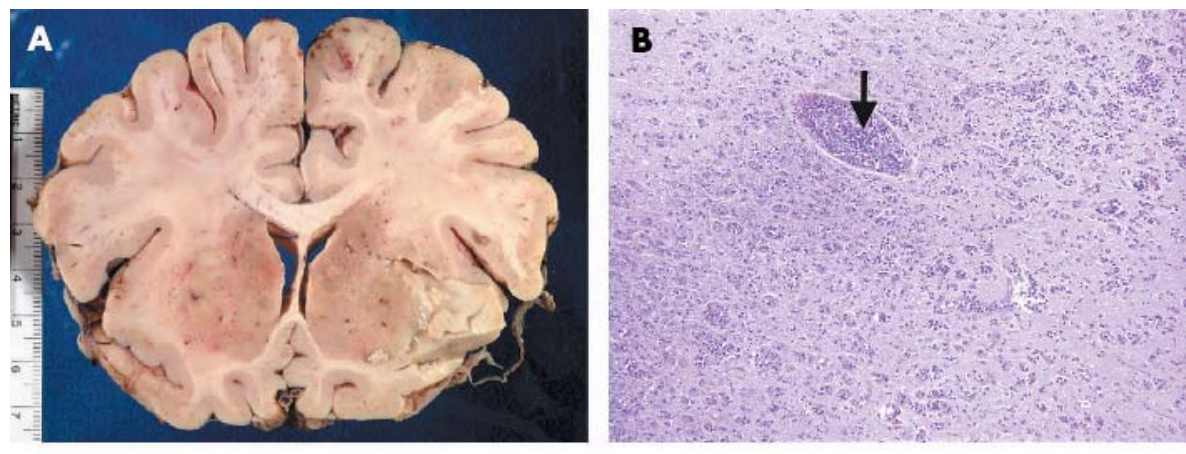

Figure 2 (A) Coronal section of brain showing infarction of basal ganglia and multiple cortical infarcts. (B) Occlusion of small vessel in infarcted area with acute inflammatory reaction (arrow) (H\&E stain). (C) Fibrinoid necrosis of vessel in area of infarction (arrow). (D) Medulla with area of demyelination (arrow) (Luxol fast blue/H\&E counter stain) with insets showing axonal injury demonstrated by immunoperoxidase stain for neurofilaments (upper) and macrophage reaction with immunoperoxidase stain PG-MI (lower).
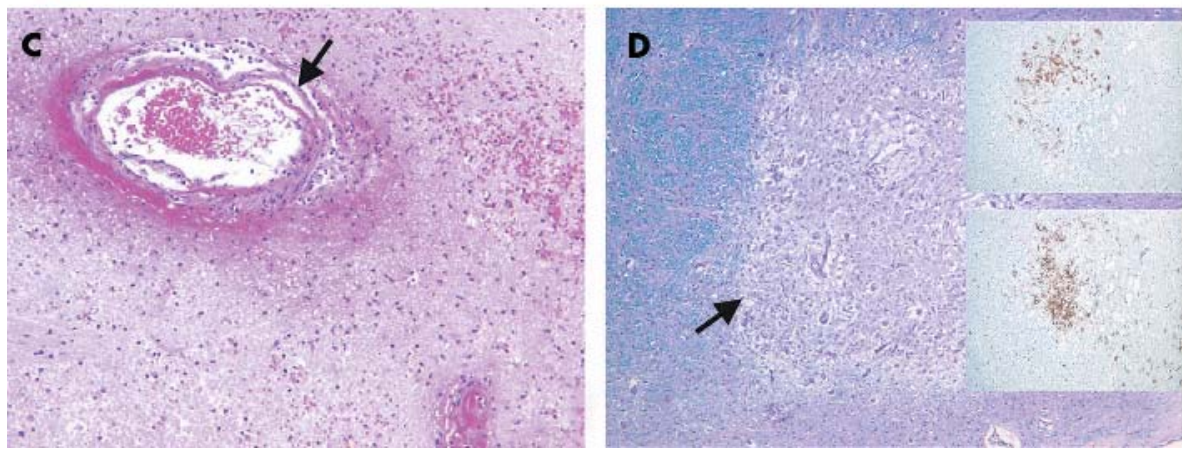
findings in our patient were at first attributed to hypoxicischaemic events; however the hypoxia and hypotension that followed both seizures prior to intubation were mild, transient, and not associated with persistent change in the patient's baseline clinical condition. Further, the neuroimaging abnormalities exceed MRI changes described following prolonged cardiac arrest in patients with more profound cerebral anoxia in the same time period (early subacute phase). ${ }^{67}$

Neuropathological findings described in association with sepsis include astrocyte and microglia proliferation, microabscesses, central pontine myelinolysis, cerebral oedema, purpuric lesions, and non-specific findings; one-third of autopsies showed no evidence of microabscesses. ${ }^{28}$ The significance of these pathological findings is not clear, and their relationship to SE is uncertain, because the sepsis had been present for weeks and complicating medical issues were frequently present. ${ }^{8}$

The MRI abnormalities in our patient correlated well with the finding of infarction of the basal ganglia, which was secondary to fibrinoid necrosis and thrombosis of small vessels. Less striking histopathological changes were appreciated in the cerebellum, which showed subtle abnormalities of minimal reactive gliosis, oedema, and acute ischaemic changes of Purkinje cells, while the medulla showed foci of demyelination with axonal swelling. Oedema and ischaemic lesions of the thalamus correlated with involvement on MRI. Despite positive immunostaining for $E$. coli in the renal abscess at autopsy, the routine and immunohistochemical stains as well as PCR of brain tissue were negative for bacteria, indicating that bacterial infection was not the direct cause of septic appearing infarctions. Immunohistochemical staining and PCR studies have not previously been reported. Our findings demonstrate that bacteria need not be present in the brain to cause pathologic features of SE. The hippocampus, a principal target and indicator of the degree of hypoxia, showed only mild involvement, supporting the concept that hypoxia-ischaemia was not the primary factor in the widespread changes seen on neuroimaging.

It is postulated that following the systemic inflammatory reaction, a cascade of events triggered by release of cytokines such as tumour necrosis factor and interleukins leads to a disturbance of cerebral microvessel endothelial cells that, in conjunction with mild hypoxia and hypotension, resulted in the extensive bilateral deep grey matter brain lesions seen, indicating that bacterial infection was not the direct cause of the septic appearing infarctions seen both on neuroimaging and at autopsy. ${ }^{13}$ Given the clinical setting, we are not aware of a similar neuroimaging pattern as reported here. ${ }^{9}$ It remains to be seen if these findings are specific for SE. Nevertheless, MRI changes of diffuse bilateral deep grey matter involvement as described here should raise suspicion for SE, particularly in the context of a critically ill patient.

\section{Authors' affiliations}

P F Finelli, D F Uphoff, Departments of Neurology and Pathology, Hartford Hospital, University of Connecticut School of Medicine, Hartford, CT, USA

Competing interests: none declared

Correspondence to: P F Finelli, MD, Hartford Hospital, 80 Seymour Street, Hartford, CT 06102-5037, USA; Pfinell@harthosp.org

Received 22 October 2003

Accepted 7 November 2003

\section{REFERENCES}

1 Powers JH, Scheld WM. Fever in neurologic diseases. Infect Dis Clin North Am 1996;10:45-66.

2 Bleck TP, Smith MC, Peirre-Louis S J-C, et al. Neurologic complications of critical medical illness. Crit Care Med 1993;21:98-103.

3 Papadopoulos MC, Davies DC, Moss RF, et al. Pathophysiology of septic encephalopathy: a review. Crit Care Med 2000;28:3019-24.

4 Llewelyn M, Cohen J. New insights into the pathogenesis and therapy of sepsis and septic shock. In: Remington JS, Swartz MN, eds. Current Clinical Topics in Infectious Diseases. USA: Blackwell Science, 2001;21:148-71.

5 Höllinger P, Zürcher R, Schroth $G$, et al. Diffusion magnetic resonance imaging findings in cerebritis and brain abscesses in a patient with septic encephalopathy. J Neurol 2000;247:232-4.

6 Arbelaez A, Castillo M, Mukherii SK. Diffusion-weighted MR imaging of global cerebral anoxia. AJNR Am J Neuroradiol 1999;20:999-1007.

7 Wijdicks EFM, Campeau NG, Miller GM. MR Imaging in comatose survivors of cardiac resuscitation. AJNR Am J Neuroradiol 2001;22:1561-5.

8 Bolton CF, Young GB. Neurological complications in critically ill patients. In: Aminoff MJ, ed. Neurology and General Medicine, 2nd edn. New York: Churchill Livingstone, 1995:859.

9 Finelli PF, DiMario FJ. Diagnostic approach in patients with symmetric imaging lesions of the deep grey nuclei. Neurologist 2003;9:250-61. 